\title{
AVALIAÇÃO CARDIORRESPIRATÓRIA DE PACIENTES ONCOLÓGICOS COM FADIGA SUBMETIDOS A UM PROTOCOLO DE ENDURANCE NA ESTEIRA
}

\author{
CARDIORRESPIRATORY EVALUATION OF ONCOLOGICAL \\ PATIENTS WITH FATIGUE SUBMITTED TO AN \\ ENDURANCE PROTOCOL IN THE MAT
}

Ana Paula Rocha Nepomuceno Edson (Orcid: 0000-0002-9903-5515) (1,2,3 $^{1,3}$

Vanessa Mendes de Lima (Orcid: 0000-0003-4199-9562) 2,4

Gisele Alves de Sousa Assunção (Orcid: 0000-0002-9750-4058)2

Bárbara Beatriz Joei Mioto (Orcid: 0000-0002-7590-5475) ${ }^{2}$

Camilla Vaticola dos Santos (Orcid: 0000-0001-6227-0303) 2,4

\begin{abstract}
RESUMO
O câncer é uma enfermidade considerada um problema de saúde pública que requer um tratamento dispendioso, além de gerar efeitos sistêmicos no paciente, sendo a fadiga o sintoma mais presente durante e depois da terapia adjuvante. Objetivo: avaliar o impacto de um protocolo de endurance na capacidade cardiorrespiratória de pacientes oncológicos com fadiga. Materiais e métodos: o estudo foi realizado com 13 pacientes oncológicos em quimioterapia e/ou radioterapia com fadiga, sendo um ensaio clínico não controlado. Os instrumentos de avaliação foram: escala de Piper Revisada, espirometria, teste de caminhada de 6 minutos (TC-6) e cirtometria. O protocolo iniciou em $60 \%$ da distância obtida no TC6, sendo acrescentado $10 \%$ da distância alcançada em cada atendimento até o quinto. A partir do sexto, $5 \%$ do valor da distância obtida no último atendimento foi acrescentado até o décimo. O protocolo foi aplicado em dez atendimentos de dez minutos, com média de três atendimentos semanais, em um total de quatro semanas. A análise estatística foi realizada usando o software IBM SPSS Statistics (v.21.0). As variáveis foram descritas por meio de mediana, primeiro e terceiro quartil (1Q - 3Q), sendo adotado o nível de significância de 5\% ( $\alpha=$ 0,05). Os dados foram comparados por meio do teste não paramétricos de Wilcoxon. Para analisar a correlação das variáveis, foi aplicado o teste de Spearman. Resultados: constatou-se uma redução no nível de fadiga, aumento mediano de aproximadamente $1,0 \mathrm{~km} / \mathrm{h}$ nas velocidades inicial, final e máxima alcançada, como também um aumento mediano de $178 \mathrm{~m}$ da distância percorrida no décimo minuto, além da melhora dos parâmetros da espirometria e da cirtometria; todos estes apresentaram significância estatística entre pré e pós-intervenção. Conclusão: os pacientes oncológicos do estudo obtiveram melhora significativa na capacidade cardiorrespiratória e redução dos níveis de fadiga, apesar de fatores como peso, estadiamento clínico e alterações fisiológicas gerados pelo tratamento.

Palavras-chave: Fadiga; Neoplasia maligna; Terapia combinada; Modalidades de Fisioterapia; Exercício.
\end{abstract}

Contato

Ana Paula Rocha Nepomuceno Edson E-mail: paula.aprn@gmail.com

${ }^{1}$ Serviço de Fisioterapia, Universidade Federal do Ceará, Ceará, Brasil. ${ }^{2}$ Serviço de Fisioterapia, Hospital Erasto Gaertner, Paraná, Brasil.

${ }^{3}$ Irmandade Beneficiente Santa Casa de Misericórdia de Fortaleza, Ceará, Brasil.

${ }^{4}$ Hospital Universitário Evangélico de Curitiba, Paraná, Brasil.

\begin{abstract}
Cancer is a disease considered a public health problem that requires expensive treatment, in addition to generating systemic effects in the patient, with fatigue being the most common symptom during and after adjuvant therapy. Objective: to evaluate the impact of an endurance protocol on the cardiorespiratory capacity of cancer patients with fatigue. Materials and methods: the study was carried out with 13 oncologic patients with fatigue under chemotherapy and/or radiotherapy, being it an uncontrolled clinical trial. The instruments of evaluation were: Piper Revised scale, spirometry, 6-minute walk test (6-MWT), and cirtometry. The protocol started in $60 \%$ of the distance obtained in the $6 \mathrm{MWT}$, being added $10 \%$ of the distance reached in each service until the fifth. From the sixth, $5 \%$ of the value of the distance obtained in the last service was added until the 10th. The protocol was applied in ten ten-minute visits, with an average of three weekly visits, in a total of four weeks. Statistical analysis was performed using the IBM SPSS Statistics software (v.21.0). The variables were described by means of median, first and third quartiles (1Q - 3Q), and the significance level of $5 \%(\alpha=0.05)$ was adopted. Data were compared using the non-parametric Wilcoxon test. To analyze the correlation of the variables, the Spearman test was applied. Results: a reduction in the fatigue level, a median increase of approximately $1.0 \mathrm{~km} / \mathrm{h}$ in the initial, final, and maximum velocities achieved, as well as a median increase of $178 \mathrm{~m}$ in the distance traveled in the tenth minute, as well as the improvement of the parameters spirometry and cirtometry; all of these presented statistical significance between pre and post-intervention. Conclusion: oncologic patients in the study obtained a significant improvement in cardiorespiratory capacity and reduction in fatigue levels, despite factors such as weight, clinical staging, and physiological alterations caused by the treatment. Keywords: Fatigue. Neoplasms. Combination modality therapy. Physical therapy modalities. Exercise.
\end{abstract}




\section{INTRODUÇÃO}

Segundo cálculos da OMS, em 2020, o número estimado de novos casos de câncer (CA) em todo o mundo alcançará 15 milhões. Hoje, essa doença é considerada a segunda causa de mortes no mundo. Atualmente, 20 milhões de indivíduos vivem com CA, e cerca de 10 milhões deles morrem a cada ano. Sua incidência tem aumentado tanto nos países desenvolvidos como naqueles em desenvolvimento, como resultado da crescente exposição a fatores de risco e do aumento da expectativa de vida ${ }^{1}$.

Entre as principais abordagens no tratamento do CA, estão a quimioterapia, a radioterapia, a hormonioterapia e a cirurgia, sendo esta última a modalidade inicial mais utilizada em vários tipos de cânceres ${ }^{2}$. A ordenação e a combinação dessas terapias dependem de múltiplos elementos, como estadiamento clínico, localização anatômica, tamanho do tumor, histologia e fatores relacionados ao paciente, tais como idade, estado geral e outros $^{3}$.

Os avanços no diagnóstico e tratamento do CA tem possibilitado uma elevação da sobrevida em um número cada vez maior de pessoas com doenças oncológicas. Muitos desses pacientes podem apresentar várias disfunções sistêmicas e incapacidades, temporárias ou permanentes. Essas ocorrências podem ser decorrentes da própria evolução da neoplasia ou como consequências associadas a doenças crônicas preexistentes ${ }^{4}$.
Segundo o Instituto Nacional do Câncer dos Estados Unidos, 72\% a 95\% dos pacientes com CA, que recebem tratamento, apresentam aumento nos níveis de fadiga, resultando em diminuição da capacidade funcional e consequente perda da qualidade de vida ${ }^{5}$.

O metabolismo de pacientes com neoplasia maligna sofre alterações extremas em virtude do estresse pelo tratamento ou pela própria doença, o que pode acarretar depressão psicológica, ansiedade, diminuição do apetite, perda de massa muscular, astenia, deficit de equilíbrio, dispneia e até caquexia ${ }^{6}$. O exercício físico pode minimizar esses efeitos ao reduzir a fadiga, melhorar a capacidade cardiorrespiratória e a qualidade de vida dos pacientes em tratamento oncológico, mesmo diante de evidências de doença ativa ou não ${ }^{7,8}$.

A fadiga se caracteriza como um dos sintomas mais debilitantes e crônicos do paciente oncológico, sendo observada durante e depois do tratamento adjuvante. Alguns pacientes sofrem com essa sintomatologia por meses e até anos após os tratamentos realizados ${ }^{9,10}$. Estudos comprovaram que a incorporação de exercícios físicos, tanto de alta quanto de baixa intensidade, pode reduzir os níveis de fadiga em pacientes com diversos tipos de $\mathrm{CA}^{11,12}$.

Um dos fatores relacionados a esse sintoma é o estado hipermetabólico somado a crescimento tumoral, competição entre o organismo e o tumor por nutrientes, efeitos deletérios da quimioterapia e radioterapia, ingestão 
nutricional inadequada associada à náusea, bem como vômitos decorrentes da terapêutica antineoplásica, anemia, distúrbios do sono ou estresse prolongado $^{13}$.

$\mathrm{Na}$ literatura, existem pesquisas que seguiram protocolos específicos para treino aeróbio em pacientes oncológicos e que se mostraram eficazes para diminuição da fadiga. Há estudos que evidenciam os benefícios do exercício físico na prevenção de fadiga durante o tratamento do CA.

$\mathrm{O}$ exercício físico em pacientes oncológicos pode interferir nos processos biológicos relacionados com o crescimento ou recorrência do tumor. Como consequência, constatam-se melhora na imunidade, redução da atividade inflamatória, atenuação dos efeitos metabólicos adversos da imobilidade e da quimioterapia, redução do risco de complicações cardiovasculares, tudo isso trazendo um aumento da autoestima e da qualidade de vida do paciente ${ }^{14-16}$.

Nesse contexto, um protocolo de tratamento para fadiga em pacientes oncológicos com base no TC-6 e na progressão da melhora ventilatória com treino de endurance é um método comparativo de fácil aplicação e de baixo custo. A aplicação desse protocolo tem a finalidade de reduzir a fadiga causada pelo tratamento oncológico, pois esse tratamento causa desgaste físico e mental aos pacientes, além de que a literatura ainda não compreende muitos estudos que contemplem essa população em tratamento adjuvante apresentando múltiplas variáveis.
Dessa forma, esta pesquisa teve por objetivo avaliar o impacto de um protocolo de endurance na capacidade cardiorrespiratória em pacientes oncológicos com fadiga durante $\mathrm{o}$ tratamento de quimioterapia, radioterapia ou concomitantes.

\section{MATERIAIS E MÉTODOS}

O presente estudo foi aprovado, sob aspecto de relevância clínica, pelo Comitê de Ética em Pesquisa do Hospital Erasto Gaertner (HEG), localizado na cidade de Curitiba-PR, com base na Resolução 466/2012, que regulamenta as pesquisas envolvendo seres humanos. Caracteriza-se como estudo experimental: ensaio clínico não controlado e aplicado em duas fases $\left(1^{\circ}\right.$ semestre de 2017 e $1^{\circ}$ semestre de 2018). Certificado de Apresentação de Apreciação Ética (CAAE) sob número 65149317.7.0000.0098.

\section{Participantes}

Os participantes foram pacientes oncológicos adultos admitidos no ambulatório da fisioterapia respiratória e motora do HEG, em processo de recuperação pneumo ou cinéticofuncional, que atenderam aos seguintes critérios de elegibilidade: a) apresentar diagnóstico de CA de qualquer localização e em qualquer estadiamento clínico; b) estar em quimioterapia e/ou radioterapia e; c) apresentar fadiga e escore superior a quatro na escala de Piper-revisada. 


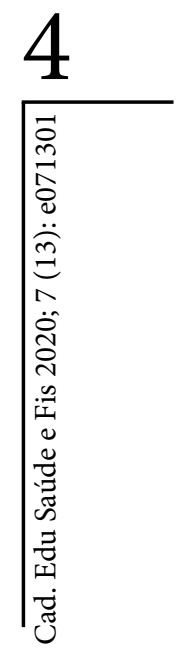

Os critérios de exclusão foram: a) estar em tratamento fisioterapêutico em outro local; b) angina instável e infarto agudo do miocárdio no mês anterior à realização do teste; c) frequência cardíaca de repouso superior a 120 batimentos por minuto; d) pressão arterial de repouso superior a $180 \mathrm{mmHg}$ (sistólica) e/ou 100 $\mathrm{mmHg}$ (diastólica); e) arritmia cardíaca não controlada; f) saturação de oxigênio basal menor que 88\%; g) desordens musculoesqueléticas que promovam marcha claudicante; $h$ ) perturbações do foro cognitivo/psiquiátrico que sejam impeditivas de garantir um grau de colaboração eficaz; i) presença de dor torácica ou abdominal; j) presença de sinais e sintomas como dispneia intensa, vômitos, hemoptise e diarreia; l) infecção respiratória em curso e; m) gravidez.

A pesquisa foi aplicada em duas fases, sendo a primeira em 2017 (abril a julho) com 5 participantes, e a segunda em 2018 (abril a junho) com 8 participantes, ou seja, ao todo, 13 participantes.

Todos os indivíduos foram devidamente informados acerca dos procedimentos a serem realizados e assinaram o Termo de Consentimento Livre e Esclarecido (TCLE), de acordo com a resolução 466/12. O estudo foi composto por doze encontros; o primeiro e o último foram as avaliações e reavaliações, com dez atendimentos de intervenções programadas.

\section{Materiais}

Para a execução desta pesquisa, foram utilizados os seguintes materiais: uma esteira, um oxímetro portátil, um espirômetro, bocais, clipe nasal, uma fita métrica.

As avaliações ocorreram da seguinte forma:

a) Ficha de identificação do paciente: perfil sociodemográfico e clínico;

b) Escala de Fadiga de Piperrevisada: usada para avaliar o nível de fadiga, composta por 23 itens subdivididos em 4 diferentes domínios: afetivo, sensorial, cognitivo e comportamental. Em cada categoria, o nível de fadiga é categorizado por meio de um escore que varia de zero a dez pontos, sendo que: zero significa ausência de fadiga; nível médio de fadiga varia de um a três pontos; nível moderado de fadiga varia de quatro a seis pontos; e nível severo de fadiga corresponde à faixa de sete a dez pontos $^{17}$;

c) Teste de caminhada de 6 minutos (TC-6): usado para calcular a distância predita, a velocidade máxima alcançada e a distância alcançada. Durante o teste, são avaliadas a frequência cardíaca e a saturação com o oxímetro portátil, a percepção do nível de esforço com a Escala de Borg modificada, essas três medidas no primeiro, terceiro e sexto minutos ${ }^{18}$; 
d) Prova de função pulmonar (espirometria): exame usado para a coleta de dados referentes ao Volume Expirado Forçado no Primeiro Segundo (VEF1), que é o volume de ar (medido em litros) exalado no primeiro segundo da manobra da capacidade vital forçada (CVF) observada durante a expiração; à relação VEF1/CVF - razão entre o volume expirado forçado no primeiro segundo e a capacidade vital forçada - usada para identificar um distúrbio obstrutivo; e à Ventilação Voluntária Máxima (VVM), que representa o volume máximo de ar ventilado em um período por repetidas manobras respiratórias forçadas. A partir desses dados, pode-se classificar o grau de restrição ou obstrução e o risco pulmonar $^{19}$;

e) Cirtometria ou perimetria tóraco-abdominal: avaliou-se a expansibilidade torácica por meio de medidas das circunferências do tórax e abdômen durante os movimentos respiratórios, utilizando o Coeficiente de Amplitude axilar (CAax), xifoidiana (CAxif) e basal ou abdominal (CAab). Foi usada uma fita métrica escalonada em centímetros horizontalmente em três níveis: axilar, xifoidiano e abdominal, em posição ortostática. Em cada ponto de referência, foram realizadas 3 inspirações e expirações máximas, sendo anotados os 3 valores $e$ as diferenças obtidas entre elas (coeficiente de amplitude) $^{20}$.
Avaliações Finais: depois de completados os 10 atendimentos, todos os participantes da pesquisa foram reavaliados por meio de todos os testes e escalas supracitados, para comparação dos dados.

\section{Intervenção}

Os participantes foram submetidos a um protocolo para treino de endurance na esteira, baseado no esforço submáximo apresentado a partir do teste de caminhada de 6 minutos (TC-6). Durante esse teste, cada paciente caminhou em velocidade sustentada na esteira, sem correr, durante seis minutos.

A frequência cardíaca (FC) e a saturação de oxigênio $\left(\mathrm{SpO}_{2}\right)$ foram monitoradas durante o teste, além do acompanhamento do nível de esforço com a Escala de Borg modificada ${ }^{17}$. Sua finalidade foi mensurar a maior distância percorrida pelo indivíduo caminhando em sua velocidade máxima durante seis minutos.

O protocolo iniciou com $60 \%$ da distância obtida no TC-6. Em seguida, foram acrescentados $10 \%$ da distância alcançada em cada atendimento, até o quinto atendimento. A partir do sexto atendimento, $5 \%$ do valor da última distância alcançada foram acrescentados até atingir os 10 atendimentos. 
O tempo de aplicação do protocolo foi de 10 minutos, a distância percorrida até o sexto minuto foi anotada e usada como parâmetro de melhora. Durante o atendimento, foram monitorizados a $\mathrm{FC}$ e $\mathrm{SpO}_{2}$ por meio de um oxímetro de Dedo Portátil AT 101C Bioland ${ }^{\circledR}$; e a percepção do nível de esforço, pela escala de Borg adaptada.

\section{Análise dos resultados}

As variáveis foram descritas por meio de mediana, primeiro e terceiro quartil (1Q - 3Q), por serem mais representativas de amostras pequenas, e um nível de significância de 5\% ( $\alpha=0,05)$, ou seja, o valor de $\mathrm{p}<0,05$ foi assumido em todas as análises. Portanto, o resultado é estatisticamente significante quando o p-valor observado é menor que o nível de significância a definido para o estudo.

As comparações entre pré e pósintervenções foram realizadas por meio do Software IBM SPSS (versão 21.0). Para verificar a distribuição dos dados, foi utilizado o teste não paramétrico de Wilcoxon $(\alpha=0,05)$. Para investigar a existência de associação entre as variáveis, foi calculado o coeficiente de correlação de Spearman.

\section{RESULTADOS}

A amostra foi composta por 13 participantes, cujas características estão apresentadas por meio de Mediana (1Q 3Q): 60,8 anos, $68 \mathrm{~kg}, 1,63 \mathrm{~m}$ de altura e IMC $21,71 \mathrm{~kg} / \mathrm{m}^{2}$.
Na tabela 1, estão apresentadas as características da amostra, sendo o grupo dividido em cinco mulheres e oito homens: três negam tabagismo, quatro são tabagistas e seis são ex-tabagistas. Quanto às comorbidades: três negam, três são diabéticos, cinco são hipertensos e dois apresentam diabetes e hipertensão arterial sistêmica.

Quanto à localização do CA: quatro apresentaram tumor de cabeça e pescoço, o mais prevalente, seguido de mama e pulmão, cada um com três participantes diagnosticados, dois apresentaram CA de próstata e um paciente era portador de tumor renal (tabela 1).

$$
\text { O estadiamento clínico está }
$$
dividido em: I e II, cada um com um paciente nesse estadio; IIIA e IIIB, dois pacientes em cada estádio; e sete deles se apresentavam no estadio IV. Quanto ao tratamento, seis pacientes estavam somente em quimioterapia, seis estavam em terapia combinada de radioterapia e quimioterapia e um deles, em radioterapia e hormonioterapia (tabela 1).

O grau de risco pulmonar dos pacientes foi avaliado por meio da espirometria e classificado da seguinte forma: dois apresentaram grau II, seis obtiveram grau III na prova e cinco tiveram como resultado grau IV. No gráfico 1, o grau de risco pulmonar dos pacientes apresenta-se em porcentagem: $15,38 \%$ apresentou grau II, $46,15 \%$ obteve grau III na prova e $38,46 \%$ teve como resultado grau IV. 
Tabela 1. Caracterização dos participantes da pesquisa. Curitiba, 2018.

\begin{tabular}{|c|c|c|}
\hline Variáveis & $\mathbf{n}$ & $\%$ \\
\hline \multicolumn{3}{|l|}{ Gênero } \\
\hline Feminino & 5 & 38,5 \\
\hline Masculino & 8 & 61,5 \\
\hline \multicolumn{3}{|l|}{ Tabagismo } \\
\hline Sim & 4 & 30,8 \\
\hline Não & 3 & 23,1 \\
\hline Ex & 6 & 46,2 \\
\hline \multicolumn{3}{|l|}{ Comorbidades } \\
\hline Nega & 3 & 23,1 \\
\hline $\mathrm{DM}$ & 3 & 23,1 \\
\hline HAS & 5 & 38,5 \\
\hline $\mathrm{DM}+\mathrm{HAS}$ & 2 & 15,4 \\
\hline \multicolumn{3}{|l|}{ Local do câncer } \\
\hline Mama & 3 & 23,1 \\
\hline Próstata & 2 & 15,4 \\
\hline Pulmão & 3 & 23,1 \\
\hline Rim & 1 & 7,7 \\
\hline Cabeça e pescoço & 4 & 30,8 \\
\hline \multicolumn{3}{|l|}{ Estadiamento clínico } \\
\hline IIIA & 2 & 15,4 \\
\hline IV & 7 & 53,8 \\
\hline II & 1 & 7,7 \\
\hline IIIB & 2 & 15,4 \\
\hline I & 1 & 7,7 \\
\hline \multicolumn{3}{|l|}{ Tratamento } \\
\hline Qt & 6 & 46,2 \\
\hline $\mathrm{Rxt}+\mathrm{Qt}$ & 6 & 46,2 \\
\hline $\mathrm{Rxt}+\mathrm{Ht}$ & 1 & 7,7 \\
\hline
\end{tabular}

Qt: quimioterapia; Ht: hormonioterapia; Rxt: radioterapia; HAS: hipertensão arterial sistêmica; DM: diabetes mellitus. 


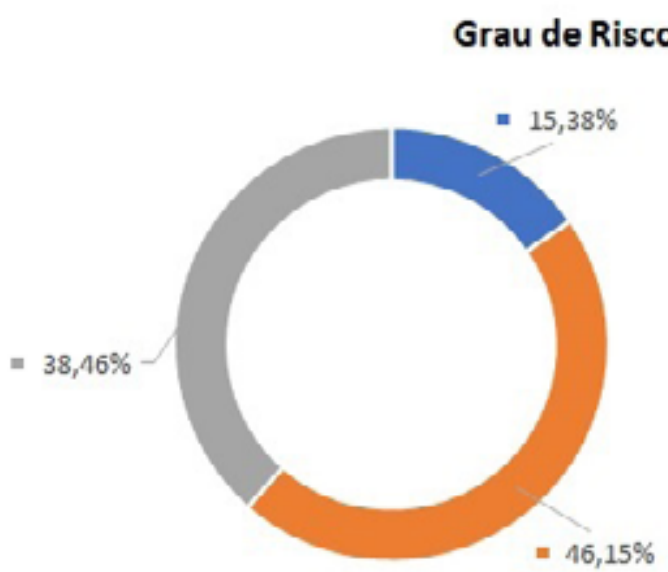

Os valores de mediana (1Q 3Q) das variáveis avaliadas durante a realização do protocolo de endurance no $1^{\circ}$ e no $10^{\circ}$ atendimento, bem como os valores de $\mathrm{p}$ das comparações, estão apresentados na tabela 2 .

Diferenças com significância estatística foram observadas para as seguintes variáveis: velocidades inicial, final e máxima alcançada, com aumento mediano de aproximadamente $1,0 \mathrm{~km} / \mathrm{h}$ cada; FC no primeiro minuto com mediana entre 81 bpm e 85 bpm; SpO2 com mediana acima de $93 \%$ no primeiro, quinto e décimo minutos; distância percorrida no décimo minuto, com aumento mediano de $178 \mathrm{~m}$. As demais variáveis não apresentaram diferenças estatisticamente significantes.

$\mathrm{Na}$ tabela 3, estão apresentados os valores medianos (1Q - 3Q) do TC-6 nos períodos de pré e pós-intervenção: distância percorrida com aumento de $65 \mathrm{~m}$ conforme a mediana e velocidade máxima alcançada com aumento de 1,25 $\mathrm{km} / \mathrm{h}$. Ambos os resultados mostraram significância estatística.
- Grau de Risco Pulmonar II - 15,38\%

- Grau de Risco Pulmonar III - 46,15\%

- Grau de Risco Pulmonar IV - $38,46 \%$
Os valores medianos (1Q - 3Q) da escala de Piper nos períodos de pré e pós-intervenção mostram que os níveis de fadiga de Piper foram menores após as intervenções, e essa diferença tem significância estatística (tabela 3).

Na tabela 4, estão apresentados os valores medianos (1Q - 3Q) da prova de função pulmonar nos períodos de pré e pós-intervenção: VEF1, CVF, VEF1/ CVF e VVM respectivamente. Na mesma tabela, estão apresentados os valores medianos (1Q - 3Q) da cirtometria nos períodos de pré e pós-intervenção: axilar, xifoidiana e abdominal respectivamente.

Os valores aumentaram entre os períodos de pré e pós-intervenção, CVF e VEF1/CVF com aumento de $4 \%$. Apesar de ocorrer uma leve queda no VEF1 e do VVM de 0,5\% na mediana do pós-intervenção, todos apresentaram significância estatística.

Todas as variáveis apresentaram significância estatística, sobretudo em relação ao CAxif com $\mathrm{p}=0,004$, pois os dados apresentaram distribuição mais similares dentro do intervalo de confiança de $95 \%$. 


\begin{tabular}{|c|c|c|c|}
\hline \multirow{2}{*}{ Variáveis do protocolo } & $1^{\circ}$ Atendimento & $10^{\circ}$ Atendimento & \multirow{2}{*}{ Valor de $p$} \\
\hline & Mediana (1Q - 3Q) & Mediana $(1 Q-3 Q)$ & \\
\hline \multicolumn{4}{|l|}{$1^{\circ} \mathrm{Min}$} \\
\hline $\mathrm{FC}(\mathrm{bpm})$ & $85(53-117)$ & $81(63-99)$ & $0,034^{*}$ \\
\hline $\mathrm{SpO}_{2}(\%)$ & $94(92-96)$ & $93,5(89-98)$ & $0,004^{*}$ \\
\hline Borg & $0,25(0,0-0,5)$ & $0,0(0,0-0,0)$ & 1,41 \\
\hline \multicolumn{4}{|l|}{$5^{\circ}$ Min } \\
\hline $\mathrm{FC}(\mathrm{bpm})$ & $81(59-103)$ & $115(101-130)$ & 0,173 \\
\hline $\mathrm{SpO} 2(\%)$ & $93,5(90-97)$ & $94(91-97)$ & $0,004^{*}$ \\
\hline Borg & $0,5(0,0-1,0)$ & $1,25(0,5-2,0)$ & 0,428 \\
\hline \multicolumn{4}{|l|}{$10^{\circ}$ Min } \\
\hline FC (bpm) & $87(60-114)$ & $98(71-125)$ & 0,059 \\
\hline $\mathrm{SpO} 2(\%)$ & $94,5(92-97)$ & $95,5(93-98)$ & $0,007^{\star}$ \\
\hline Borg & $1,5(1,0-2,0)$ & $2,75(2,5-3,0)$ & 0,415 \\
\hline \multicolumn{4}{|l|}{ Velocidade $(\mathbf{k m} / \mathbf{h})$} \\
\hline Inicial & $3,25(2,5-4,0)$ & $4,3(3,6-5,0)$ & $0,049^{*}$ \\
\hline Final & $4,25(2,0-6,5)$ & $5,05(4,0-6,1)$ & $0,043^{\star}$ \\
\hline Máxima alcançada & $4,25(2,0-6,5)$ & $5,1(4,0-6,2)$ & $0,044^{*}$ \\
\hline \multicolumn{4}{|l|}{ Distância percorrida } \\
\hline No $6^{\circ} \min (\mathrm{m})$ & $375(210-540)$ & $438(330-545)$ & 0,076 \\
\hline No $10^{\circ} \min (\mathrm{m})$ & $592(375-810)$ & $770(655-885)$ & $0,047^{\star}$ \\
\hline
\end{tabular}

FC: frequência cardíaca; bpm: batimentos por minuto; Min: minuto; SpO2: saturação periférica de oxigênio; km/h: quilômetros por hora; m: metros; * Significância estatística (Wilcoxon $\alpha=0,05$ ).

Tabela 3. Resultados do Teste de Caminhada dos seis minutos e da escala de Piper Revisada. Curitiba, 2018.

\begin{tabular}{lccc}
\hline \multirow{2}{*}{ Variáveis } & Pré-intervenção & Pós-intervenção & \multirow{2}{*}{ Valor de $\boldsymbol{p}$} \\
\cline { 2 - 3 } & Mediana $(\mathbf{1 Q}-\mathbf{3 Q})$ & Mediana (1Q - 3Q) & $0,048^{\star}$ \\
\hline TC6 & $370(210-530)$ & $435(330-540)$ & $0,049^{\star}$ \\
\hline $\begin{array}{l}\text { Distância percorrida }(\mathrm{m}) \\
\text { Velocidade máxima }\end{array}$ & $3,8(2,5-5,1)$ & $5,05(4,0-6,1)$ & \\
alcançada $(\mathrm{km} / \mathrm{h})$ & & & $0,035^{*}$ \\
\hline Escala de Piper Revisada & $5,0(4,5-5,5)$ & $2,5(1,0-4,0)$ & \\
\hline Fadiga & & & \\
\hline
\end{tabular}

$\mathrm{km} / \mathrm{h}$ : quilômetros por hora; m: metros; ${ }^{\star}$ Significância estatística (Wilcoxon $\alpha=0,05$ ). 


\begin{tabular}{|c|c|c|c|}
\hline \multirow[b]{2}{*}{ Variáveis } & Pré-intervenção & Pós-intervenção & \multirow[b]{2}{*}{ Valor de $p$} \\
\hline & Mediana (1Q - 3Q) & Mediana (1Q - 3Q) & \\
\hline \multicolumn{4}{|l|}{ Prova de função pulmonar } \\
\hline VEF1 (\%) & $67,50(44-91)$ & $67,00(47-87)$ & $0,022^{*}$ \\
\hline CVF (\%) & $64(40-88)$ & $68(44-92)$ & $0,042^{*}$ \\
\hline VEF1/CVF (\%) & $80(53-90)$ & $84,50(60-94)$ & $0,038^{*}$ \\
\hline VVM (\%) & $62(38-86)$ & $61,50(36-87)$ & $0,022^{*}$ \\
\hline \multicolumn{4}{|c|}{ Coeficiente de amplitude Axilar (CAax) } \\
\hline Repouso (cm) & $96,5(85-102)$ & $98(87-104)$ & $0,012^{*}$ \\
\hline Inspiração Máxima (cm) & $98(93-103)$ & $96,5(91-102)$ & $0,011^{*}$ \\
\hline Expiração Máxima (cm) & $94(89-99)$ & $92(86-98)$ & $0,015^{*}$ \\
\hline \multicolumn{4}{|c|}{ Coeficiente de amplitude Xifoidiana (CAxif) } \\
\hline Repouso (cm) & $89,5(75-104)$ & $90(78-102)$ & $0,004^{*}$ \\
\hline Inspiração Máxima (cm) & $95,5(84-107)$ & $97(88-106)$ & $0,011^{\star}$ \\
\hline Expiração Máxima (cm) & $88(79-97)$ & $86(77-95)$ & $0,016^{*}$ \\
\hline \multicolumn{4}{|c|}{ Coeficiente de amplitude Abdominal (CAabd) } \\
\hline Repouso (cm) & $86,5(73-100)$ & $88,5(77-100)$ & $0,016^{*}$ \\
\hline Inspiração Máxima (cm) & $93(84-102)$ & $91(80-102)$ & $0,015^{*}$ \\
\hline Expiração Máxima (cm) & $88(79-97)$ & $86(77-95)$ & $0,016^{*}$ \\
\hline
\end{tabular}

VEF1: Volume Expiratório Forçado no $1^{\circ}$ segundo; CVF: Capacidade Vital Forçada; VVM: Ventilação Voluntária Máxima; cm: centímetros. $\left.{ }^{\star S i g n i f i c a ̂ n c i a ~ e s t a t i ́ s t i c a ~(W i l c o x o n ~} a=0,05\right)$.

Tabela 5. Correlação entre o teste de caminhada dos seis minutos e demais variáveis do estudo. Curitiba, 2018.

\begin{tabular}{|c|c|c|c|c|}
\hline \multirow{3}{*}{ Variáveis } & \multicolumn{2}{|c|}{ TC -6} & \multicolumn{2}{|c|}{ TC - 6} \\
\hline & \multicolumn{2}{|c|}{ Distância Percorrida } & \multicolumn{2}{|c|}{ Velocidade Máxima Alcançada } \\
\hline & rho & $p$ & rho & $p$ \\
\hline \multicolumn{5}{|l|}{ Escala de Piper Revisada } \\
\hline Fadiga & $-0,392$ & 0,221 & $-0,548$ & 0,236 \\
\hline \multicolumn{5}{|l|}{ Função Pulmonar } \\
\hline CVF & 0,952 & $0,049^{*}$ & 0,629 & 0,102 \\
\hline VVM & $-0,752$ & 0,524 & $-0,687$ & 0,537 \\
\hline \multicolumn{5}{|l|}{ Coeficiente de Amplitude } \\
\hline Axilar em repouso & 0,657 & 0,842 & 0,492 & 0,200 \\
\hline Axilar Insp. Máx. & $-0,658$ & 0,881 & $-0,195$ & 0,897 \\
\hline Axilar Exp. Máx. & $-0,903$ & 0,075 & $-0,248$ & 0,753 \\
\hline Xifoidiana em repouso & 0,572 & 0,196 & 0,689 & 0,120 \\
\hline Xifoidiana Insp. Máx. & 0,697 & 0,886 & 0,825 & 0,089 \\
\hline Xifoidiana Exp. Máx. & $-0,358$ & 0,707 & $-0,265$ & 0,717 \\
\hline
\end{tabular}

TC-6: Teste de Caminhada de Seis minutos; CVF: Capacidade Vital Forçada; VVM: Ventilação Voluntária Máxima; Insp. Máx: inspiração máxima; Exp. Máx: expiração máxima; rho: Coeficiente de Correlação de Spearman; *Significância estatística no nível de 5\%. 
Na tabela 5, estão apresentados os resultados da associação entre as variáveis do TC-6 (distância percorrida e velocidade máxima alcançada) com os resultados da escala de Piper, da prova de função pulmonar (CVF e VVM) e da cirtometria (CAax e CAxif). Essas variáveis foram escolhidas para associação entre si, por contemplarem os tópicos de avaliação do estudo. Além disso, entre os resultados com significância estatística, esses podem ser os mais representativos.

Entre as variáveis que apresentaram associações significativas na correlação de Spearman, estão: a distância percorrida no TC-6 com a CVF da prova de função pulmonar e o CAax na expiração máxima; a velocidade máxima alcançada no TC-6 com o CAxif. Nas três correlações destacadas, os valores do coeficiente foram altos, sendo dois positivos e um negativo. A correlação entre distância percorrida no TC-6 com a CVF da prova de função pulmonar foi a única a apresentar significância estatística com $\mathrm{p}=0,049$.

\section{DISCUSSÃO}

Após a aplicação do protocolo de treinamento de endurance na esteira em pacientes oncológicos com fadiga, verificaram-se a redução dos níveis de fadiga, a melhora nos resultados da prova de função pulmonar e do TC-6, além da significância encontrada entre as comparações da cirtometria.

Participaram do estudo 13 pacientes em terapia adjuvante - oito homens e cinco mulheres -, cujas características foram dadas por Mediana (1Q - 3Q): 60,8 anos, $68 \mathrm{~kg}, 1,63 \mathrm{~m}$ de altura e IMC $21,71 \mathrm{~kg} / \mathrm{m}^{2}$. Entre eles, três negam tabagismo, quatro são tabagistas e seis são ex-tabagistas. Quanto às comorbidades: três negam, três são diabéticos, cinco são hipertensos e dois apresentam diabetes e hipertensão arterial sistêmica concomitantes.

De acordo com os dados, há predominância no estudo de homens (61,5\%), ex-tabagistas (46,15\%) e hipertensos (38,46\%), corroborando o estudo de Pereira et al., que avaliou 94 idosos internados no Hospital Universitário da Universidade Federal do Pará, com a média de idade de 71,57 $( \pm 8,06)$ anos, sendo que, desses, apenas sete tinham diagnóstico fechado de CA, a maioria formada por homens $(57,14 \%)$ e por hipertensos $(57,14 \%)^{21}$.

Quanto à localização do CA, no presente estudo, quatro participantes apresentaram tumor de cabeça e pescoço, seguido de mama e pulmão, cada um com três participantes diagnosticados, dois com CA de próstata e um paciente com tumor renal.

Já no estudo de Pereira et al., o perfil epidemiológico é composto por dois casos de CA no pâncreas, dois casos de CA no estômago, um caso de CA no fígado, um caso de mieloma múltiplo e um caso de CA no esôfago, todos associados a, pelo menos, outra doença crônica, como hipertensão arterial, diabetes mellitus e cirrose hepática $^{21}$.

Essa diferença de perfil epidemiológico se dá pela composição de costumes e hábitos entre as regiões do Brasil, uma vez que o presente estudo foi realizado na região Sul, e o estudo de Pereira et al., na região Norte. 
O estadiamento clínico está dividido em: I e II, cada um com um paciente, IIIA e IIIB, dois pacientes em cada estadio e sete deles se apresentavam no estadio IV. Quanto ao tratamento, seis pacientes estavam somente em quimioterapia, seis em terapia combinada de radioterapia e quimioterapia e um deles em radioterapia e hormonioterapia.

$\mathrm{Na}$ revisão de Courneya ${ }^{22}$, entre os estudos considerados, houve uma prevalência dos ensaios clínicos randomizados feitos com pacientes portadores de CA de mama (6 dos 11 estudos consultados). A maioria dos pacientes desses estudos se encontrava nos estadios II e III, como também em quimioterapia. Esse predomínio de pesquisas com pacientes de CA de mama em estadios II e III expressa a situação mais comum para a adjuvância com quimioterapia para redução do tumor e uma posterior cirurgia.

Diferenças com significância estatística foram observadas no TC-6 para as seguintes variáveis: velocidades inicial, final e máxima alcançada, com aumento mediano de aproximadamente $1,0 \mathrm{~km} / \mathrm{h}$ cada; FC no primeiro minuto com mediana entre 81 bpm e 85 bpm; $\mathrm{SpO} 2$ com mediana acima de $93 \%$ no primeiro, quinto e décimo minutos; distância percorrida no décimo minuto, com aumento mediano de $178 \mathrm{~m}$.

Cesario et al. ${ }^{23}$ observaram no TC-6 um aumento na média das distâncias percorridas de 95,6 m após um programa de exercícios em pacientes internados para o tratamento do CA de pulmão. Nesse estudo, foram comparados os grupos de intervenção e controle antes do programa, observando-se que o grupo controle percorreu uma distância significativamente maior $(p=0,01)$. Essa diferença não teve significância após a realização de 20 sessões de exercício, tornando os dois grupos mais homogêneos.

A distância final percorrida durante os 10 atendimentos foi de $65 \%$ a $80 \%$ maior do que a distância máxima encontrada no TC-6, conforme a tolerância de cada participante. Esse achado corrobora os resultados encontrados no estudo realizado por Courneya e colaboradores ${ }^{24}$, o qual utilizou a intensidade moderada de $60 \%$ a $70 \%$ durante o protocolo de endurance na esteira com 53 mulheres com CA de mama, três vezes por semana, por 15 semanas ${ }^{24}$.

Nos períodos de pré e pósintervenção do TC-6, foram evidenciados: distância percorrida com aumento de $65 \mathrm{~m}$ conforme a mediana e velocidade máxima alcançada com aumento de 1,25 $\mathrm{km} / \mathrm{h}$. Ambos os resultados mostraram significância estatística.

Quist et al. avaliaram a capacidade funcional dos pacientes com neoplasia pulmonar no pré e pósintervenção, utilizando como métodos de avaliação o TC-6, os testes incrementais e a ergoespirometria - por meio da medida do consumo máximo de oxigênio (Vo2máx) e/ou do trabalho (W). Eles também identificaram ganho significativo após a aplicação do protocolo ${ }^{25}$. 
O presente estudo também utilizou dados da prova de função pulmonar conhecida como espirometria, nos períodos de pré e pós-intervenção. Os valores finais de CVF e VEF1/CVF aumentaram em $4 \%$, apesar de ocorrer uma leve queda no VEF1 e no VVM de $0,5 \%$ na mediana do pós intervenção. Todas essas variáveis apresentaram significância estatística.

Schneider et al. realizaram um estudo investigando as alterações da função cardiopulmonar e fadiga, por meio de um protocolo com exercícios individualizados e alternados entre aeróbicos e de força/endurance por 6 meses, em 45 homens sobreviventes de CA. Também utilizaram a prova de função pulmonar com espirometria, mas a comparação entre o pré e pósintervenção não resultou em aumento com significância estatística nas variáveis CVF e VEF1, apesar da melhora na função cardiopulmonar ${ }^{26}$.

Os valores medianos (1Q 3Q) da escala de Piper nos períodos de pré e pós-intervenção apresentaram diminuição dos níveis de fadiga de Piper no pós-intervenção, e essa diferença foi significativa.

Schneider et al. também avaliaram, além da capacidade cardiorrespiratória, o nível de fadiga antes e após o programa de exercícios em pacientes com diferentes diagnósticos de CA. O protocolo consistiu em uma combinação de treino aeróbico e não aeróbico, resultando na redução significativa dos sintomas de fadiga dos participantes em relação a todas as concepções de fadiga exploradas no estudo $^{26}$.

No estudo randomizado e controlado feito por Markes, Brockow e Resch, foram avaliadas 119 pacientes com CA de mama durante quimioterapia ou radioterapia, com média de idade de 51 anos. Desse total, 11 não concluíram o protocolo. As participantes receberam uma prescrição para fazer caminhada de moderada intensidade, cinco a seis vezes por semana, com frequência cardíaca de $50 \%$ a $70 \%$ da frequência cardíaca máxima ${ }^{27}$.

$\mathrm{O}$ protocolo consistiu em uma caminhada rápida de 15 minutos, progredindo para 30 minutos. Esse programa foi realizado durante o tratamento, seis semanas de radioterapia ou três a seis meses de quimioterapia. Como resultado, o exercício físico aeróbico domiciliar reduziu de forma estatisticamente significativa $\quad(\mathrm{p}=0,03)$ os sintomas de fadiga em pacientes com CA de mama durante o tratamento ${ }^{27}$. O presente estudo obteve resultados similares na atenuação da fadiga, utilizando a escala de Fadiga de Piperrevisada.

Todas as variáveis apresentaram significância estatística, sobretudo em relação ao CAxif com $\mathrm{p}=0,004$, pois os dados apresentaram distribuição mais similares dentro do intervalo de confiança de $95 \%$. 
Bezerra, Santos-Júnior e Campos avaliaram a relação dos CAax, CAxif e CAab em 50 adultos jovens utilizando a cirtometria. Quando comparado à expansibilidade entre os sexos, não houve diferença estatisticamente significante ( $\mathrm{p}=0,6878)$, mas, ao comparar as diferenças entre os locais de aferição, estes se mostraram diferentes entre si $(\mathrm{p}=0,0019)^{20}$.

Levou-se em consideração os resultados da associação entre as variáveis do TC-6 (distância percorrida e velocidade máxima alcançada) e os resultados da escala de Piper, da espirometria (CVF e VVM) e da cirtometria (CAax e CAxif), pois as variáveis que apresentaram associações significativas na correlação de Spearman são as seguintes: a distância percorrida no TC-6 com a CVF da espirometria e o CAax na expiração máxima; e a velocidade máxima alcançada no TC-6 com o CAxif.

A correlação entre distância percorrida no TC-6 com a CVF da prova de função pulmonar foi a única a apresentar significância estatística com $\mathrm{p}=0,049$. Não foram encontrados estudos que avaliassem as associações e correlações apresentadas nos dois últimos parágrafos, mas o estudo abaixo apresenta resultados referentes à fadiga, variável pertinente a esta pesquisa, além de condicionamento cardiovascular e funcionamento físico sob a percepção do paciente, também relevantes.

Courneya et al. realizaram um ensaio randomizado controlado em que foram avaliados 122 pacientes com linfoma, sendo que o grupo de intervenção com 60 pacientes foi submetido a exercícios aeróbicos supervisionados por 12 semanas $^{28}$.
Os autores avaliaram adesão, funcionamento físico avaliado pelo paciente, fadiga, qualidade de vida geral, felicidade, depressão, saúde geral, condicionamento cardiovascular e massa corporal magra. A mudança no pico de aptidão mediou o funcionamento físico avaliado pelo paciente ${ }^{28}$.

Não houve interferência no grupo de intervenção na resposta ao tratamento ou na taxa de conclusão da quimioterapia e no seguimento de seis meses. O grupo de intervenção teve resultados superiores aos do grupo controle em qualidade de vida geral, felicidade e redução da depressão, sem um risco aumentado de progressão da doença ${ }^{28}$.

Um problema bastante frequente nos diferentes estudos com pacientes oncológicos, e enfrentado pelas pesquisadoras neste estudo, é a falta de adesão, mesmo que os benefícios dos programas de exercícios já estejam bem estabelecidos. Esse fato se deve à característica dos protocolos de treinamento, que exigem, no mínimo, algumas semanas de assiduidade. Entre os motivos que levam à evasão, enumeramse: a hospitalização, a deterioração clínica após a quimioterapia, a dificuldade de transporte e a falta de interesse decorrente da apatia ${ }^{29}$.

Após a comparação de protocolos e resultados de vários estudos, associados a exercícios com tempo de duração similares ou diferentes, nas distintas fases de tratamento e neoplasias, observaram-se semelhanças com os resultados deste estudo. 
O presente estudo, mesmo com suas limitações, utilizando uma pequena amostra $(n=13)$ e sem grupo-controle, alcançou resultados significativos. Contudo, sugere-se realizar o mesmo estudo de endurance com um número maior de participantes para reafirmar sua efetividade, além de estudos comparativos com outros exercícios aeróbicos.

\section{REFERÊNCIAS}

1. Instituto Nacional do Câncer (INCA). Ministério da Saúde. Brasil lidera definição de políticas de prevenção do câncer para América Latina e Caribe - Incidência de Câncer no Brasil. Rio de Janeiro: INCA, 2018. [Internet]. [Acessado 2018 jul 22]. Disponível em http://www.inca.gov.br/ releases/press_ release_view_arq.asp? $I D=1603$.

2. Johnston PG, Spence RAJ. Oncologia. Rio de Janeiro: Guanabara Koogan; 2003.

3. Irish J, O'Sullivan B, Siu L, Lee A. Câncer de cabeça e pescoço. In: Pollock RE, organizadores. Manual de Oncologia Clínica da UICC. Fundação Onconcentro de São Paulo. 8. ed. São Paulo: Atheneu; 2006. p. 331-354.

4. Almeida EMP, Andrade RG, Cecatto RB, Brito CMM, Camargo FP, Pinto $\mathrm{CA}$, et al. Exercício em pacientes oncológicos: reabilitação. Acta Fisiatr 2012; 19(2):82-89.
5. National Cancer Institute (NCI). Fatigue PDQ - Health Professional Version. USA.gov: August 2017. [Internet]. [Acessado 2018 jul 10]. Disponível em http://www.cancer.gov/ about-cancer/treatment/sideeffects/ fatigue/fatigue-hp-pdq.

6. Miaskowski C, Dodd M, West C, Schumacher K, Paul SM, Tripathy D, et al. Randomized clinical trial of the effectiveness of a self-care intervention to improve cancer pain management. J Clin Oncol 2004; 22(9):1713-1720.

7. Seixas RJ, Basso AGO, Marx, AG. Exercício físico aeróbico e câncer de pulmão: um estudo de revisão, 2011. Rev. Brasileira De Cancerologia [Internet] 2012 [Acessado 2018 jul 18]; 58(2):267-275. Disponível em https:// rbc.inca.gov.br/revista/ index.php/ revista/article/view/629

8. Oldervoll LM, Kassa S, Hjermstad MJ, Lund JA, Loge JH. Physical exercise results in the improved subjective wellbeing of a few or is effective rehabilitation for all cancer patients?. Eur J Cancer 2004; 40(7):951-962.

9. Adamsen L, Quist M, Andersen C, Moller T, Herrstedt J, Kronborg D, et al. Effect of a multimodal high intensity exercise intervention in cancer patients undergoing chemotherapy: randomised controlled trial. BMJ [Internet] 2009 [Acessado 2018 jul 16]; 339:b3410. Disponível em https://www.bmj.com/ content/339/bmj.b3410 
10. Cheville AL, Girardi J, Clark MM, Rummans TA, Pittelkow T, Brown P, et al. Therapeutic exercise during outpatient radiation therapy for advanced cancer: Feasibility and impact on physical well-being. Am J Phys Med Rehabil 2010; 89(8):611-619.

11. Galvão DA, Taaffe DR, Spry N, Joseph D, Newton RU. Combined resistance and aerobic exercise program reverses muscle loss in men undergoing androgen suppression therapy for prostate cancer without bone metastases: a randomized controlled trial. J Clin Oncol 2010; 28(2):340-347.

12. Amorim JR, Silva IA, Shimizu IS. Avaliação da qualidade de sono em pacientes com câncer de mama em quimioterapia. Rev Bras Mastologia 2017; 27(1):3-7.

13. Menezes MFB, Camargo TC. A Fadiga relacionada ao câncer como temática na enfermagem oncológica. Rev Latino-Am Enfermagem 2006; 14(3):442-447.

14. Temel JS, Greer JA, Goldberg S, Vogel PD, Sullivan M, Pirl WF, et al. A structured exercise program for patients with advanced non-small cell lung cancer. J Thorac Oncol 2009; 4(5):595-601.

15. Nikander R, Sievanen H, Ojala K, Oivanen T, Kellokumpu-Lehtinen PL, Saarto T. Effect of a vigorous aerobic regimen on physical performance in breast cancer patients - a randomized controlled pilot trial. Acta Oncol 2007; 46(2):181-186.

16. Spence RR, Heesch KC, Brown WJ. Exercise and cancer rehabilitation: a systematic review. Cancer Treat Rev 2010; 36(2):185-194.
17. Mansano-SchlosserI TC, Ceolim MF. Fadiga em idosos em tratamento quimioterápico. Rev Bras Enfermagem 2014; 67(4):623629. Disponível em http://www.scielo. br/scielo.phppid=S0034716720140 00400623\&script=sci_arttext\&tlng $=$ pt

18. Rondelli RR, Oliveira AN, Dal Corso S, Malaguti C. Uma atualização e proposta de padronização do Teste de Caminhada dos seis minutos. Fisioterapia em Movimento 2009; abr/jun; 22;(2):249-259.

19. Pereira CACP. Espirometria. J Pneumol 2002; 28(Supl. 3):1-82.

20. Bezerra RO, Santos-Junior FFU, Campos NG. Análise da mobilidade da caixa torácica e da força muscular respiratória em adultos jovens. EFD Esportes [Internet] 2012 [Acessado 2018 jul 12]; 17(175). Disponível em https:// www.efdeportes.com/efd175/analise-damobilidade -da-caixa-toracica.htm

21. Pereira EEB, Santos NB, Sarges ESNF. Avaliação da capacidade funcional do paciente oncogeriátrico hospitalizado. Rev Pan-Amaz Saúde 2014; 5(4):37-44.

22. Courneya KS. Exercise interventions during cancer treatment: biopsychosocial outcomes. Exerc Sport Sci Rev 2001; 29(2):60-64.

23. Cesario A, Ferri L, Galetta D, Pasqua F, Bonassi S, Clini E, et al. Post-operative respiratory rehabilitation after lung resection for non-small cell lung cancer. Lung Cancer 2007; 57(2):175-80. 
24. Courneya KS, Segal RJ, Mackey JR, Gelmon K, Reid RD, Friedenreich CM, et al. Effects of aerobic and resistance exercise in breast cancer patients receiving adjuvant chemotherapy: a multicenter randomized controlled trial. J Clin Oncol 2007; 25(28):43964404.

25. Quist M, Rørth M, Langer S, Jones LW, Laursen JH, Pappot H, et al. Safety and feasibility of a combined exercise intervention for inoperable lung cancer patients undergoing chemotherapy: a pilot study. Lung Cancer 2012; 75(2):203-208.

26. Schneider CM, Hsieh CC, Sprod LK, Carter SD, Hayward R. Exercise training manages cardiopulmonary function and fatigue during and following cancer treatment in male cancer survivors. Integr Cancer Ther 2007; 6(3):235-241.
27. Markes M, Brockow T, Resch KL. Exercise for women receiving adjuvant therapy for breast cancer. Cochrane Database Syst Rev 2006; (4): CD005001.

28. Courneya KS, Sellar CM, Stevinson C, McNeely ML, Peddle CJ, Friedenreich $\mathrm{CM}$, et al. Randomized controlled trial of the effects of aerobic exercise on physical functioning and quality of life in lymphoma patients. J Clin Oncol 2009; 27(27):4605-4612.

29. Battaglini CL, Hackney AC, Garcia R, Groff D, Evans E, Shea T. The effects of an exercise program in leukemia patients. Integr Cancer Ther 2009; 8(2):130-138. 